\title{
Escape to Nasal Cavity During Swallowing
}

National Cancer Institute

\section{Source}

National Cancer Institute. Escape to Nasal Cavity During Swallowing. NCI Thesaurus.

Code C127229.

A finding of escape to nasal cavity during swallowing. 